\title{
Pleural fluid glucose testing using a finger stick glucometer: a novel bedside test
}

\author{
Tony F. Abdo, Himanshu Bhardwaj, Muhammad K. Ishaq, Jean I. Keddissi, Houssein A. Youness \\ Interventional Pulmonary Program, Section of Pulmonary, Critical Care and Sleep Medicine, Oklahoma City VA Health Care System and University \\ of Oklahoma Health Sciences Center, Oklahoma, USA \\ Contributions: (I) Conception and design: HA Youness; (II) Administrative support: None; (III) Provision of study materials or patients: None; (IV) \\ Collection and assembly of data: TF Abdo, H Bhardwaj, MK Ishaq; (V) Data analysis and interpretation: All authors; (VI) Manuscript writing: All \\ authors; (VII) Final approval of manuscript: All authors. \\ Correspondence to: Houssein A. Youness, MD. 800 Stanton L Young Blvd, Suite 8400, Oklahoma City, Oklahoma 73104, USA. \\ Email: Houssein-Youness@ouhsc.edu.
}

\begin{abstract}
Background: Pleural fluid glucose (PFG) has diagnostic and therapeutic implications for the management of pleural effusion. The literature examining point-of-care testing of PFG is limited, and no studies exist for the bedside measurement of PFG using a glucometer (B-PFG). In this study, we compared the accuracy of B-PFG measurement to standard in-lab measurement (Lab-PFG).

Methods: Patients undergoing thoracentesis or thoracostomy were enrolled. PFG was measured at the bedside with a finger stick blood glucometer (ACCU-CHEK ${ }^{\circledR}$ Inform II, Roche) and in the laboratory. Two consecutive measurements were taken using the glucometer, and their mean was compared to the glucose concentration measured in the laboratory. Pearson correlation coefficient and Bland-Altman Plot analysis were used to compare the two measurements.

Results: Sixty patients were included. Mean age was 64.1 years. Forty-nine patients had exudative effusions (41\% malignant, $26 \%$ parapneumonic, and 33\% others). There was a significant correlation between the B-PFG and the Lab-PFG ( $r=0.98$, 95\% CI of 0.97 to $0.99 ; \mathrm{P}<0.0001)$. There was good agreement between the B-PFG and the Lab-PFG with a mean difference of $14.8 \mathrm{mg} / \mathrm{dL}$ [95\% limit of agreement (LOA) of -2.2 to $31.8 \mathrm{mg} / \mathrm{dL}$ ]. This agreement was even better at glucose values less than $80 \mathrm{mg} / \mathrm{dL}$.

Conclusions: PFG measured at the bedside with a glucometer closely correlates with the laboratory measurement. Further studies are needed prior to incorporating this test in clinical practice.
\end{abstract}

Keywords: Pleural effusion; pleural fluid glucose (PFG); glucometer; thoracentesis.

Submitted May 29, 2019. Accepted for publication Aug 19, 2019.

doi: $10.21037 /$ jtd.2019.09.04

View this article at: http://dx.doi.org/10.21037/jtd.2019.09.04

\section{Introduction}

Parapneumonic pleural effusion (PPE) is the most common exudative effusion, and the second most common effusion overall, with heart failure related effusion coming first $(1,2)$. PPE incidence has been increasing, and it carries high morbidity and mortality especially among the elderly $(3,4)$. Early identification of complicated parapneumonic effusion (CPPE) is critical since treatment with antibiotics alone is not enough, and chest tube drainage is required.

Determining the cause of a pleural effusion is greatly enhanced by analysis of the pleural fluid. Amongst the various pleural fluid tests, pleural fluid glucose (PFG) is used extensively to further narrow down the possible underlying causes. A low PFG (less than $60 \mathrm{mg} / \mathrm{dL}$ ), narrows the differential diagnosis of the exudative pleural effusion to include CPPE or empyema, malignant effusion, rheumatoid pleurisy, tuberculous pleurisy, lupus pleuritis and esophageal rupture (5). Besides, for patients with parapneumonic effusions, a low PFG has diagnostic and therapeutic implications. Both the American College of 
Chest Physicians (ACCP) and the British Thoracic Society (BTS) released guidelines for the identification of CPPE. PFG constitutes one of the identifying criteria, with levels less than $60 \mathrm{mg} / \mathrm{dL}$ and less than $40 \mathrm{mg} / \mathrm{dL}$ being consistent with CPPE, per the ACCP (6) and the BTS $(7,8)$ respectively.

The availability of PFG at the bedside may allow early identification of CPPE, and therefore earlier treatment with chest tube drainage. The literature examining point-of-care testing of PFG is limited, and no studies exist for the bedside measurement of PFG using a glucometer (B-PFG). This study aims to look into the potential accuracy and benefit of using a glucometer to measure PFG at the bedside.

\section{Methods}

In a prospective observational study, we enrolled patients from the Oklahoma City VA Health Care System and the University of Oklahoma Medical Center. The protocol was approved by the Institutional Review Board (IRB) of the University of Oklahoma Health Sciences Center (IRB\# 3785). The trial was registered in clinicaltrials.gov (NCT02208895). An informed consent was obtained from all patients.

Inclusion criteria included patients of 18-99 years old with pleural effusion requiring thoracentesis or chest tube insertion. The inability to obtain an informed consent or obtain pleural fluid during thoracentesis was the only exclusion criteria.

Data collected at the time of enrollment included age, sex, ethnicity, body mass index (BMI), medical comorbidities, and effusion side. Pleural fluid characteristics including chemical and biochemical analysis, cytology, and microbiology were recorded. Pleural fluid $\mathrm{pH}$ measurement was performed in the laboratory using a blood gas analyzer (Siemens Rapidpoint 500).

PFG concentration was measured at the bedside with a finger stick blood glucometer (ACCU-CHEK ${ }^{\circledR}$ Inform II, Roche) and the UniCel ${ }^{\circledR}$ DxC 800 Synchron $^{\circledR}$ Clinical System was used for Lab-PFG measurement. The ACCUCHEK $^{\circledR}$ Inform II glucometer was calibrated every $24 \mathrm{~h}$ as per manufacturer standards. To evaluate the intra-assay precision of the glucometer, two consecutive measurements were obtained using the glucometer and compared to each other. Pleural effusions were classified into transudates and exudates based on Light's criteria (9).

The primary outcome was the correlation between B-PFG and Lab-PFG. Secondary outcomes included the intra-assay precision of B-PFG and the elapsed time between the B-PFG and the corresponding Lab-PFG. Glucometer PFG data was not shared with the treating physician.

\section{Statistical analysis}

Statistical analyses were performed using MedCalc for Windows, version 16.0 (MedCalc Software, Ostend, Belgium). The mean of two B-PFG measurements was compared to the Lab-PFG. Pearson Correlation Coefficient and Bland-Altman Plot (10) were used for that comparison. Since the glucometer has a reportable range of 25 to $600 \mathrm{mg} / \mathrm{dL}$, PFG concentration of $25 \mathrm{mg} / \mathrm{dL}$ was used for all "undetectable low reading" on the glucometer.

\section{Results}

Sixty-one patients were enrolled. One patient did not have his laboratory glucose value measured. The data for sixty patients was analyzed subsequently. The baseline characteristics of all patients are listed in Table 1. The mean age was $64.1 \pm 11.7$ years (range, 35-92). Comorbidities included congestive heart failure (28\%), pneumonia (30\%), chronic obstructive pulmonary disease (28\%), lung cancer $(23 \%)$, other malignancies (30\%), end-stage renal disease $(12 \%)$, and liver cirrhosis (3\%). Forty-nine patients $(82 \%)$ had an exudative effusions (41\% malignant, $26 \%$ parapneumonic, and $33 \%$ others).

The average laboratory and glucometer PFG measurements were $106.6 \pm 44.4$ and $121.4 \pm 48.3 \mathrm{mg} / \mathrm{dL}$ respectively. The mean difference between the two PFG concentrations measured by the glucometer was $3 \mathrm{mg} / \mathrm{dL}$ [95\% limit of agreement (LOA) of -2.1 to $8.2 \mathrm{mg} / \mathrm{dL}$ ] suggesting a good intra-assay precision. There was a significant correlation between the B-PFG and the lab-PFG $(\mathrm{r}=0.98,95 \%$ CI of 0.97-0.99; $\mathrm{P}<0.0001$ ) (Figure $1 A$ ).

Bland-Altman plot of the mean B-PFG and the Lab-PFG value showed a strong correlation with a mean difference of $14.8 \mathrm{mg} / \mathrm{dL}$ ( $95 \% \mathrm{LOA}$ of -2.2 to $31.8 \mathrm{mg} / \mathrm{dL}$ ). A better agreement was noted at lab-PFG values of less than $80 \mathrm{mg} / \mathrm{dL}$ with a mean difference of $9.4 \mathrm{mg} / \mathrm{dL}$ (95\% LOA of -1.2 to $20 \mathrm{mg} / \mathrm{dL}$ ) (Figure 1B).

The glucometer values were available earlier than the laboratory values by a mean difference of $107 \pm 65.5$ minutes (median of 91 minutes, range of 26 to 388 minutes).

The median laboratory $\mathrm{pH}$ of all patients was 7.51 (range, 5.3-8.5). In the subgroup of patients with labPFG $<60 \mathrm{mg} / \mathrm{dL}, 50 \%(3 / 6)$ had a laboratory $\mathrm{pH}>7.2$ with a median value of 7.34 (range, 5.3-7.61). The bedside glucometer was able to identify all six pleural effusions 
Table 1 Demographic data and pleural effusion etiologies

\begin{tabular}{|c|c|}
\hline Characteristic & No. (\%), $n=60$ \\
\hline Age, mean (SD), years & $64.1(11.7)$ \\
\hline Range, years & $35-92$ \\
\hline Mean BMI & 26 \\
\hline Sex, male (\%) & $49(82 \%)$ \\
\hline Pleural effusion side, right (\%) & $34(57 \%)$ \\
\hline \multicolumn{2}{|l|}{ Medical comorbidities } \\
\hline Congestive heart failure (CHF) & $17(28 \%)$ \\
\hline Pneumonia & $18(30 \%)$ \\
\hline Chronic obstructive pulmonary disease & $17(28 \%)$ \\
\hline Lung cancer & $14(23 \%)$ \\
\hline Other malignancies & $18(30 \%)$ \\
\hline End stage renal disease (ESRD) & $7(12 \%)$ \\
\hline Liver cirrhosis & $2(3 \%)$ \\
\hline \multicolumn{2}{|l|}{ Pleural effusion etiologies } \\
\hline Transudate & $11(18 \%)$ \\
\hline Exudate & $49(82 \%)$ \\
\hline Parapneumonic & 13 \\
\hline Malignant & 20 \\
\hline Others* & 16 \\
\hline Pleural fluid glucose $<60 \mathrm{mg} / \mathrm{dL}$ & 6 \\
\hline Complicated parapneumonic & 4 \\
\hline Hemothorax & 1 \\
\hline Rheumatoid arthritis (RA) & 1 \\
\hline
\end{tabular}

*, included effusions related to CHF, ESRD, RA, hemothorax, eosinophilic pneumonia, pancreato-pleural fistula, and nonspecific pleuritis. BMI, body mass index.

with a lab-PFG $<60 \mathrm{mg} / \mathrm{dL}$. In the subgroup of patients with a laboratory $\mathrm{pH}<7.2,50 \%$ (2/4) had a laboratory and glucometer $\mathrm{PFG}>60 \mathrm{mg} / \mathrm{dL}$.

\section{Discussion}

The ACCP considers a non-purulent parapneumonic effusion as complicated if any of the following criteria are met: pleural fluid $\mathrm{pH}<7.2, \mathrm{PFG}<60 \mathrm{mg} / \mathrm{dL}$, a positive culture, or if the effusion occupies more than half of the hemithorax (6). The BTS, on the other hand, considers a pleural fluid $\mathrm{pH}<7.2$, glucose $<40 \mathrm{mg} / \mathrm{dL}, \mathrm{LDH}$
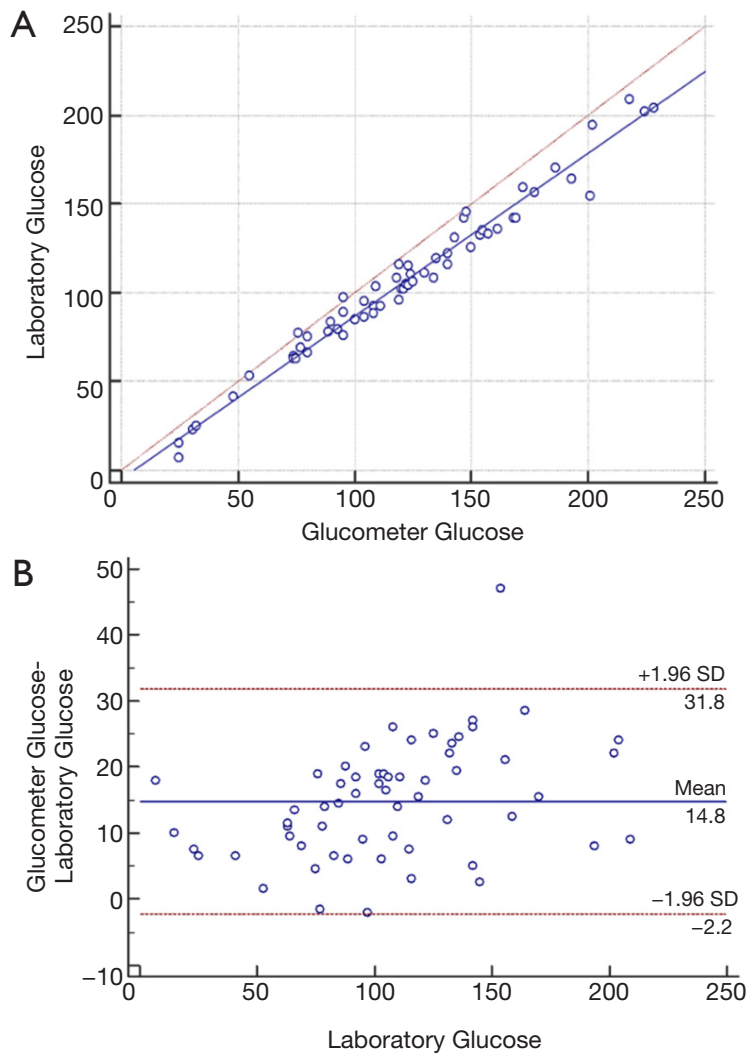

Figure 1 Comparison of pleural glucose measurement in the laboratory to the measurement by the bedside glucometer. (A) Correlation between pleural glucose measured in the laboratory and by the bedside glucometer ( $r=0.98,95 \%$ CI: 0.97-0.99; $\mathrm{P}<0.0001)$. (B) Bland-Altman plot analysis of the difference between PFG measured by the glucometer and the one measured in the laboratory showing a mean difference of $14.8 \mathrm{mg} / \mathrm{dL}(95 \%$ LOA: -2.2 to $31.8 \mathrm{mg} / \mathrm{dL}$ ). PFG, pleural fluid glucose; LOA, limit of agreement.

$>1,000 \mathrm{IU} / \mathrm{L}$, or positive culture as defining criteria for CPPE $(7,8,11)$. The pleural fluid $\mathrm{pH}$ is the most used criterion $(2,6,8)$, but multiple reports revealed that its accuracy is highly dependent on pleural fluid sample collection and measurement methods. Rahman et al. found that residual air, lidocaine, and analysis delay significantly alter the pleural fluid $\mathrm{pH}$ and may impact clinical management. On the other hand, PFG was not significantly affected by these factors (12). Furthermore, it is recommended that a blood gas analyzer should be utilized for accurate measurement of pleural fluid $\mathrm{pH}$ (13). However, nearly $30-50 \%$ of the US laboratories use other methods for pleural fluid $\mathrm{pH}$ measurement (14), and close 
to $50 \%$ of pulmonologists either did not know that a blood gas analyzer should be used, or presumed that their laboratory is using one when it is not $(14,15)$.

Despite showing that pleural fluid $\mathrm{pH}$ measured with a handheld unit (I-STAT) correlates well with the corresponding laboratory blood gas analyzer (16), many institutions would not allow testing of the pleural fluid in these units as it has not been extensively validated and it may void the manufacturer warranty. For all these reasons, PFG constituted an important parameter to consider, in addition to pleural $\mathrm{pH}$, in identifying CPPE.

Our study is the first to measure the PFG at the bedside using a glucometer. We tested a wide range (7 to $209 \mathrm{mg} / \mathrm{dL}$ ) of PFG concentrations in different pleural effusion etiologies, and a strong correlation was present throughout.

The ACCU-CHEK ${ }^{\circledR}$ Inform II, Roche glucometer is currently approved for serum glucose measurement only. When compared to the laboratory reference results, $95 \%$ of the individual serum glucose were within $\pm 15 \mathrm{mg} / \mathrm{dL}$ at glucose concentrations less than $75 \mathrm{mg} / \mathrm{dL}$ and within $\pm 20 \%$ at glucose concentrations greater than or equal to $75 \mathrm{mg} / \mathrm{dL}$ (17). Interestingly, this is slightly different from what we found using this device to measure PFG. In 58 out of 60 pleural fluid samples, B-PFG was consistently higher than the Lab-PFG with a mean difference of $14.8 \mathrm{mg} / \mathrm{dL}$ (Figure 1B). That difference was even lower (mean of $9.4 \mathrm{mg} / \mathrm{dL}$ ) when Lab-PFG is $<80 \mathrm{mg} / \mathrm{dL}$. A lower hematocrit has been associated with a higher blood glucometer reading (18); this may explain the higher B-PFG reading observed when compared to the Lab-PFG since pleural fluid hematocrit is significantly lower than the blood hematocrit. For clinical use at the bedside, B-PFG value $<70 \mathrm{mg} / \mathrm{dL}$ should be considered abnormal, and B-PFG $<60 \mathrm{mg} / \mathrm{dL}$ virtually guarantees a low Lab-PFG $<60 \mathrm{mg} / \mathrm{dL}$.

The bedside glucometer was able to identify all six pleural effusions with a lab-PFG $<60 \mathrm{mg} / \mathrm{dL}$. Remarkably, $50 \%(3 / 6)$ of these effusions had a laboratory $\mathrm{pH}$ value above 7.2 with a median value of 7.34 (range, 5.3-7.61).

Bedside PFG concentration should not be interpreted in isolation, but always in conjunction with the patient's clinical presentation. Other causes of low-glucose pleural effusions, including malignancy (19), rheumatoid arthritis, tuberculosis, and lupus, should be taken into consideration when interpreting bedside testing $(5,9,20)$.

Our study shows that when using the bedside glucometer, PFG results are obtained sooner than laboratory values by a median duration of 91 minutes. In the right clinical setting, this may allow the provider to identify CPPE immediately and proceed with chest tube insertion rather than waiting until the laboratory PFG is available. This may decrease the need to have an initial diagnostic thoracentesis followed by a chest tube placement later on.

To note that during this study, we have not shared the glucometer PFG data with the treating physician who ultimately made the decision to proceed with either a thoracentesis or an upfront chest tube placement. Overall, eighteen patients (30\%) had an initial diagnosis of pneumonia. Nine of 18 patients had evidence of either loculation or air present on the CT chest and received upfront chest tube. An additional four had upfront chest tube placement with a final diagnosis of malignant effusion in two, non complicated parapneumonic in one, and transudative effusion in another patient. Five of 18 patients underwent initial thoracentesis with one ultimately requiring chest tube for empyema. Overall, the use of bedside glucometer would have changed the management in $3 / 18(17 \%)$ patients (i.e., avoiding chest tube insertion in 2 patients and earlier placement of chest tube in one patient).

Our study has several limitations. First, it is a single center study. Second, the results obtained by this specific glucometer (ACCU-CHEK ${ }^{\circledR}$ Inform II, Roche) may not be generalized to other types of glucometers since different glucometer results can vary significantly and the agreement among them is poor (21). Third, bedside measurement may be mainly relevant to PPE. In our study, PPE was suspected in eighteen patients (30\%) (Table 1). Since this is the first study measuring PFG concentration with a glucometer, we decided not to limit it to parapneumonic effusion. We tested a wide range ( 7 to $209 \mathrm{mg} / \mathrm{dL}$ ) of PFG concentrations in different pleural effusion etiologies, and a good correlation was present throughout, with a better agreement observed at lower glucose values.

The main strengths of this study are its practical design and applicability to clinical practice. It would have been much easier to test the accuracy of the glucometer by measuring PFG concentration on every pleural fluid sample sent to the laboratory and comparing the results to standard testing. However, our goal was to test the pleural fluid with the glucometer at the bedside to simulate the intended method of use in clinical practice. Our results confirm the feasibility of this approach.

\section{Conclusions}

In conclusion, we present the first study to test PFG concentration at the bedside using a glucometer. The 
accuracy, reliability, and availability of the glucometer, make it an excellent test that can be easily integrated into clinical practice. In the right clinical setting, this method offers valuable information that may affect the diagnosis and the management of pleural effusion. Our study results may need to be validated in a larger prospective multicenter trial, using different types of glucometers.

\section{Acknowledgments}

Funding: Funding was provided partially by the Oklahoma City Veteran Affairs Health Care System.

\section{Footnote}

Conflicts of Interest: Prior Abstract Presentation-Presented at the American Thoracic Society meeting in San Diego, USA, on 5/21/2018. Role of Sponsor-Oklahoma City Veteran Affairs Health Care System provided ACCUCHECK Inform II test strips and control solutions.

Etbical Statement: The authors are accountable for all aspects of the work in ensuring that questions related to the accuracy or integrity of any part of the work are appropriately investigated and resolved. The protocol was approved by the Institutional Review Board (IRB) of the University of Oklahoma Health Sciences Center (IRB\# 3785). An informed consent was obtained from all patients.

\section{References}

1. Light RW. Clinical practice. Pleural effusion. N Engl J Med 2002;346:1971-7.

2. Feller-Kopman D, Light R. Pleural Disease. N Engl J Med 2018;378:740-51.

3. Grijalva CG, Zhu Y, Nuorti JP, et al. Emergence of parapneumonic empyema in the USA. Thorax 2011;66:663-8.

4. Hamm H, Light RW. Parapneumonic effusion and empyema. Eur Respir J 1997;10:1150-6.

5. Sahn SA. Pathogenesis and clinical features of diseases associated with a low pleural fluid glucose. Chretien J, Bignon J, Hirsch A, editors. The pleura in health and disease. New York: Marcel Dekker, 1985:267-85.

6. Colice GL, Curtis A, Deslauriers J, et al. Medical and surgical treatment of parapneumonic effusions : an evidence-based guideline. Chest 2000;118:1158-71.

7. Davies CW, Gleeson FV, Davies RJ. BTS guidelines for the management of pleural infection. Thorax 2003;58
Suppl 2:ii18-28.

8. Davies HE, Davies RJ, Davies CW. Management of pleural infection in adults: British Thoracic Society Pleural Disease Guideline 2010. Thorax 2010;65 Suppl 2:ii41-53.

9. Light RW, Macgregor MI, Luchsinger PC, et al. Pleural effusions: the diagnostic separation of transudates and exudates. Ann Intern Med 1972;77:507-13.

10. Bland JM, Altman DG. Statistical methods for assessing agreement between two methods of clinical measurement. Lancet 1986;1:307-10.

11. Hooper C, Lee YC, Maskell N. Investigation of a unilateral pleural effusion in adults: British Thoracic Society Pleural Disease Guideline 2010. Thorax 2010;65 Suppl 2:ii4-17.

12. Rahman NM, Mishra EK, Davies HE, et al. Clinically important factors influencing the diagnostic measurement of pleural fluid $\mathrm{pH}$ and glucose. Am J Respir Crit Care Med 2008;178:483-90.

13. Cheng DS, Rodriguez RM, Rogers J, et al. Comparison of pleural fluid $\mathrm{pH}$ values obtained using blood gas machine, $\mathrm{pH}$ meter, and pH indicator strip. Chest 1998;114:1368-72.

14. Putnam B, Elahi A, Bowling MR. Do we measure pleural fluid pH correctly? Curr Opin Pulm Med 2013;19:357-61.

15. Bowling $M$, Lenz $P$, Chatterjee A, et al. Perception versus reality: the measuring of pleural fluid $\mathrm{pH}$ in the United States. Respiration 2012;83:316-22.

16. Kohn GL, Hardie WD. Measuring pleural fluid pH: high correlation of a handheld unit to a traditional tabletop blood gas analyzer. Chest 2000;118:1626-9.

17. ACCU-CHEK Inform II System Evaluation Report. Indianapolis, IN. Roche Diagnostics Corporation 2012.

18. Ramljak S, Lock JP, Schipper C, et al. Hematocrit interference of blood glucose meters for patient selfmeasurement. J Diabetes Sci Technol 2013;7:179-89.

19. Rodriguez-Panadero F, Lopez Mejias J. Low glucose and $\mathrm{pH}$ levels in malignant pleural effusions. Diagnostic significance and prognostic value in respect to pleurodesis. Am Rev Respir Dis 1989;139:663-7.

20. Sahn SA. State of the art. The pleura. Am Rev Respir Dis 1988;138:184-234.

21. Kimberly MM, Vesper HW, Caudill SP, et al. Variability among five over-the-counter blood glucose monitors. Clin Chim Acta 2006;364:292-7.

Cite this article as: Abdo TF, Bhardwaj H, Ishaq MK, Keddissi JI, Youness HA. Pleural fluid glucose testing using a finger stick glucometer: a novel bedside test. J Thorac Dis 2019;11(11):49044908. doi: $10.21037 /$ jtd.2019.09.04 